\title{
Précision des mesures de vitesse de croissance des streptocoques lactiques dans le lait basées sur la méthode de dénombrement microbien par formation de colonies. Étude de référence avec Lactococcus lactis
}

\author{
A.I. Hassan, N. Deschamps et J. Richard \\ INRA, station de recherches laitières, 78350 Jouy-en-Josas, France \\ (reçu le 20 décembre 1988, accepté le 10 mai 1989)
}

\begin{abstract}
Résumé - Une souche protéolytique (prt+) de Lactococcus lactis subsp. lactis et un variant non protéolytique (prt ${ }^{-}$) de cette souche ont été cultivés dans du lait écrémé reconstitué. L'expérience a été répétée à 6 reprises à 1 ou 2 semaines d'intervalle, en respectant scrupuleusement le même protocole. A partir des courbes de croissance de chaque souche $\left(\log _{10}\right.$ des unités formant colonies en fonction du temps) on a délimité des intervalles de temps pendant lesquels la culture paraissait en phase exponentielle de croissance. Par analyse de régression des résultats sur ces intervalles, on a déterminé la vitesse de multiplication de ces souches. Par ailleurs, on a établi la validité de la méthode de numération microbienne par formation de colonies en comparant les résultats qu'elle donne avec ceux d'une méthode de comptage cellulaire sous le microscope (méthode DEFT).

On a montré que la culture de la souche $\mathrm{prt}^{+}$comportait au moins 2 phases principales de croissance, la première (de la $1 \mathrm{re}$ à la $4^{\mathrm{e}}$ heure de culture) était réellement exponentielle; la seconde (de la $4^{e}$ à la $6^{e}$ heure) l'était moins nettement. Les courbes de croissance de la souche prt ne montraient qu'une seule phase exponentielle, de la $1^{\text {re }}$ à la $4^{e}$ heure, avec une pente moyenne ne différant pas significativement de celle de la souche prt+ dans le même intervalle de temps de culture. Cependant, l'ajustement à une droite n'était pas aussi bon qu'avec la souche $\mathrm{prt}^{+}$. On a déterminé la valeur de ces pentes et leur précision. On a montré que la précision intrinsèque des pentes dépend du modèle mathématique utilisé pour interpréter les résultats, du nombre de prélèvements effectués durant la phase exponentielle de croissance, et de la précision des dénombrements. La variabilité des mesures de vitesse de croissance, d'une expérience à l'autre, a également été estimée.

Les résultats de la présente étude, permettent de dégager des recommandations pour obtenir le maximum de précision dans la détermination de la vitesse de croissance des bactéries lactiques dans le lait.
\end{abstract}

Streptocoque lactique - lait - vitesse de croissance - précision - reproductibilité

Summary - Accuracy and precision of measurements of lactic streptococci growth rates in milk, using the colony counting technique. A reference study on Lactococcus lactis. A proteolytic strain (prt) of Lactococcus lactis subsp. lactis and one of its non proteolytic variants 
(prt $\left.{ }^{-}\right)$were cultivated separately in reconstituted skim-milk. The experiment was carried out 6 times with intervals of 1 or 2 weeks between each repetition. The same protocol was used very carefully during the study in order to estimate the reproducibility of the determinations. From the growth curves of each strain ( $\log _{10}$ of colony forming units as a function of time) intervals of time during which the growing culture seemed to be in an exponential phase of growth were selected. By regression analysis on these intervals, the growth rates of the strains were calculated. On the other hand, the validity of the enumeration method by formation of colonies was checked by comparing its results with those of the direct cell counting under the microscope (DEFT method).

The culture of the prt strain showed two main phases of growth, the first one (from the 1st up to the 4th hour of culture) was really in exponential phase, as shown by the statistical analysis of the residuals; the observed values in the second phase (from the 4th to the 6th hour), did not fit the regression line so well. The growth curves of the prt-strain showed only one exponential phase of growth, from the 1st to the 4 th hour, with an average slope which was not significantly different from that obtained with the prt+ strain in the same time interval of culture. However, the adjustment of the points to a straight line was not as good as for the prt+ strain. The intrisic precision of the slopes was shown to depend on the mathematical model used for interpreting the results, the number of samples taken during the exponential phase of growth and the precision of the counting method. The variability of the growth rates between experiments was also estimated.

lactic streptococci - milk - growth rate - precision - accuracy - reproducibility colony count technique

\section{INTRODUCTION}

La détermination précise de la vitesse de multiplication des bactéries lactiques dans le lait est d'une grande utilité pour deux raisons : d'une part, elle donne une information sur la valeur du lait comme milieu de culture pour ces bactéries; d'autre part, elle permet d'expliquer les différences de vitesse d'acidification, par la même souche, d'échantillons de lait de diverses origines, ou encore, de déterminer l'activité acidifiante spécifique d'une souche donnée (vitesse d'acidification rapportée au niveau de population bactérienne et à la vitesse de multiplication de cette flore). La méthode de choix, pour déterminer la vitesse de multiplication d'un micro-organisme dans un milieu liquide, consiste généralement à mesurer la densité optique de ce milieu. Elle est applicable à un liquide opaque comme le lait, grâce à un traitement de transparisation (Kanasaki et al., 1975; Breheny et al., 1975; Turner \& Thomas, 1975; Kouomegne et al., 1984). Malheureusement, ce traitement aboutit à une dilution de l'échantillon de lait, et par conséquent, à une augmentation du seuil de sensibilité de la méthode (valeur la plus faible mesurée avec précision) (Anonyme, 1985).

La méthode de dénombrement des micro-organismes par formation de colonies sur un milieu nutritif gélosé possède un seuil de sensibilité aussi bas qu'on le veut; en revanche, elle n'a pas une fidélité aussi grande que les méthodes analytiques, celles de la chimie, par exemple. Cela est dû à au moins deux facteurs. D'une part, on n'a pas toujours une relation étroite et constante entre le nombre de cellules microbiennes viables (c'est-à-dire capables de se multiplier et de former une colonie sur ou dans le milieu gélosé) et le nombre de colonies 
effectivement formées. En particulier, selon la nature de la flore microbienne et le mode d'agitation du lait, les colonies peuvent provenir, soit des cellules isolées, soit d'agrégats cellulaires plus ou moins importants (Tatini et al., 1967; Martley, 1972; Richard, 1980; Piton \& Richard, 1983). C'est pourquoi, par souci de rigueur, on devrait toujours exprimer les résultats de dénombrements microbiens par formation de colonies en termes d' "unités formant colonies" (ufc). Cela ne résout pas pour autant le problème de la validité de la méthode (rapport entre le nombre de colonies et le nombre total de cellules ou le nombre de cellules viables).

D'autre part, on sait depuis longtemps (Fisher et al., 1922) que le nombre de cellules microbiennes par unité de volume, ou le nombre de colonies qu'elles donnent par boîte de Petri, suit une loi de distribution statistique décrite par Poisson en 1837 : si plusieurs conditions sont respectées, dont une distribution au hasard des micro-organismes dans l'inoculum, la moyenne des dénombrements des colonies sur plusieurs boîtes de Petri donne, à la fois, une estimation de la concentration cellulaire exacte de l'inoculum, et une estimation de la variance de la distribution (ou erreur de la méthode de dénombrement). Cela veut dire par exemple, que dans de bonnes conditions d'analyse, si l'on trouve une moyenne de 50 colonies, l'écart type sera d'environ 7 colonies, soit un coefficient de variation de $14 \%$. Avec une moyenne de 300 colonies, ce coefficient est ramené à $5,8 \%$. Ce sont, de toute façon, des variations très largement supérieures à celle que l'on obtient sur les volumes d'inoculum incorporés dans la gélose, dans le cas de la méthode classique de dénombrement, ou déposés à la surface de la gélose, dans le cas d'un ensemencement avec un appareil Spiral. II résulte de ces deux particularités de la méthode de dénombrement par formation de colonies (validité douteuse et manque de fidélité), que les vitesses de croissance des streptocoques lactiques dans le lait peuvent être entachées d'une grande incertitude.

La présente étude avait pour but d'abord de déterminer la validité de la méthode de dénombrement par formation de colonies, pour la mesure de vitesse de multiplication des bactéries lactiques dans le lait. On a cherché ensuite à mettre en évidence l'influence du défaut de répétabilité de cette méthode sur la précision des mesures de vitesse de croissance des bactéries lactiques. En outre, on s'est efforcé d'évaluer le degré de variabilité des résultats d'une expérience à l'autre (leur reproductibilité), lorsque les conditions expérimentales étaient maintenues aussi constantes que possible.

\section{MATÉRIEL ET MÉTHODES}

\section{Lait de culture}

Pour cette étude, le lait était préparé de la façon suivante : $100 \mathrm{~g}$ de poudre de lait écrémé "low heat" (poudre NILAC, NIZO, Pays-Bas) étaient dispersés dans 1 I d'eau distillée. $\mathrm{Ce}$ lait reconstitué était stérilisé à l'autoclave pendant $5 \mathrm{~min}$ à $120^{\circ} \mathrm{C}$.

\section{Origine des souches et préparation des cultures}

Deux souches de Lactococcus lactis subsp. lactis (anciennement Streptococcus lactis; Anonyme, 1986) ont été utilisées : la souche CNRZ 1076, qui est protéase-positive et 
lactose-positive, et l'un de ses variants, la souche CNRZ 1075, qui est protéase-négative mais lactose-positive. Ces souches étaient conservées à moins $20^{\circ} \mathrm{C}$ sur du lait tournesolé. Après décongélation, les souches étaient cultivées une nuit à $30^{\circ} \mathrm{C}$. A partir de cette préculture, on effectuait une série de dilutions décimales dans du lait. Les tubes étaient incubés de 16 à $18 \mathrm{~h}$ à $30^{\circ} \mathrm{C}$. Le tube non coagulé suivant immédiatement le dernier tube coagulé, dans la série de dilutions, était pris pour ensemencer la culture soumise à l'étude de vitesse de croissance. On estimait ainsi que les souches étaient en phase de croissance exponentielle.

\section{Étude de la vitesse de croissance}

Pour cette étude, le lait était distribué en flacons de $200 \mathrm{ml}$. La culture choisie pour l'ensemencement de ce lait était agitée vigoureusement à l'aide d'un agitateur à turbine Ultra-Turrax (20 $000 \mathrm{rot} / \mathrm{min})$, de façon à disperser au maximum les amas cellulaires (Richard, 1980). La souche protéase-positive $\left(\mathrm{prt}^{+}\right)$était inoculée à raison de $0,1 \%$, et la souche prt - à $0,2 \%$. Le lait ainsi inoculé était incubé dans un bain-marie à $30^{\circ} \mathrm{C}$ pendant $24 \mathrm{~h}$.

L'expérience a été répétée 6 fois dans des conditions aussi constantes que possible (même lot de poudre de lait, même préparation du lait et des cultures...).

\section{Dénombrement des bactéries}

A intervalles de temps réguliers (Fig. 1), on prélevait environ $15 \mathrm{ml}$ de lait dans les flacons, après les avoir agités doucement de façon à obtenir une mise en suspension des agrégats cellulaires, tout en limitant au maximum l'incorporation d'air dans le lait. Cette prise d'essai était traitée $30 \mathrm{~s}$ à l'Ultra-Turrax, puis une dilution convenable était ensemencée à l'aide d'un appareil Spiral sur le milieu M17 (Terzaghi \& Sandine, 1975) précoulé en boîtes de Petri de $14 \mathrm{~cm}$ de diamètre. Les colonies étaient comptées visuellement, après $48 \mathrm{~h}$ d'incubation des boîtes à $30^{\circ} \mathrm{C}$.

\section{Étude de la taille des agrégats cellulaires}

Pour déterminer avec précision le nombre de cellules par agrégat ou par chaînette de cocci, nous avons utilisé la méthode DEFT (Cousins et al., 1979; Rodrigues \& Kroll, 1985). Cette méthode de coloration facilite la reconnaissance des cellules et permet aussi, dans une certaine mesure, de voir si elles sont viables ou non. Cette détermination a été faite à deux reprises, au cours de l'étude, et a porté sur tous les échantillons prélevés pour les dénombrements par comptage des colonies. Le nombre d'agrégats et le nombre de cellules par agrégat ont été relevés sur 15 champs microscopiques (facteur multiplicatif du microscope égal à 13600 ).

\section{Exploitation statistique des résultats}

On a d'abord effectué une transformation logarithmique de base 10 des nombres de $u f c / m l$, puis on a fait une représentation graphique de ces résultats en fonction du temps. On pouvait ainsi repérer un intervalle de temps durant lequel les points expérimentaux étaient relativement bien alignés. On effectuait ensuite, avec les résultats appartenant à cet intervalle, une analyse de régression linéaire comportant le calcul de la pente de la droite de régression (ce qui correspond à la vitesse de croissance de la souche, en unité de log/h), l'écart-type $s_{b}$ de cette détermination, permettant de calculer l'intervalle de confiance à $95 \%$ de la pente, et l'écart type de régression $s_{y, x}$ (Snedecor \& Cochran, 1957).

\section{RÉSULTATS ET DISCUSSION}

\section{Validité de la méthode de dénom- brement par formation de colonies}

Au cours de la culture dans le lait, les souches prt+ et prt - se présentaient sous 
le même aspect microscopique : on n'observait pratiquement que des chaînettes de deux cellules ou d'un multiple de deux. Cela se comprend aisément si l'on admet que la liaison récente entre deux cellules "filles" est plus solide que la liaison plus ancienne entre deux couples de cellules. D'autre part, toutes les cellules ayant pris la coloration orange, on peut penser qu'elles étaient aussi viables les unes que les autres (Hobbie et al., 1977), cela aussi bien après qu'avant traitement à l'Ultra-Turrax.

Avant ce traitement, on avait une majorité de chaînettes composées de diplocoques (Tableau I). Entre 1 et $4 \mathrm{~h}$ d'incubation du lait, la fréquence de ces diplocoques était significativement plus faible qu'entre 4 et $8 \mathrm{~h}$ de culture. Inversement, les chaînes de plus de deux cellules étaient moins fréquentes dans cette deuxième période de culture que dans la première. Malgré cette différence, on peut dire que les souches choisies pour la présente étude, n'avaient pas une tendance prononcée à former de longues chaînes. Ce n'est pas le cas de toutes les souches de cette espèce bactérienne (Martley, 1972).

Après la dispersion des agrégats avec I'Ultra-Turrax, la fréquence des cellules isolées et, dans une moindre mesure, celle des diplocoques, augmentait au détriment de la fréquence des chaînettes de plus de deux cellules. II n'y avait plus alors de différence entre les deux périodes de culture. Le nombre moyen de cellules par chaînette était égale à 2,2. En supposant que tout agrégat donne une colonie (donc qu'il contient au moins une cellule viable), on peut donc admettre que le nombre de ufc sous-estime de moitié environ le nombre réel de cellules présentes dans le lait. Cependant, la validité de la technique de dénombrement par formation de colonies microbiennes pour toute la durée de la culture n'est pas en cause, puisque ce rapport est constant.

Tableau I. Longueur des chaînettes formées par Lactococcus lactis subsp. lactis (souches CNRZ 1075 et 1076) au cours de leur culture à $30^{\circ} \mathrm{C}$ dans du lait écrémé reconstitué. (Méthode DEFT; moyennes de 4 déterminations : 2 souches $\times 2$ répétitions).

\begin{tabular}{lccccc}
\hline $\begin{array}{l}\text { Nombre de } \\
\text { cellules par } \\
\text { chaînette }\end{array}$ & $\begin{array}{c}\text { Dispersion } \\
\text { par } \\
\text { Ultra-Turrax }\end{array}$ & initial & $\begin{array}{c}\text { Pourcentage } \\
\text { phase 1 } \\
(1 \text { à } 4 h)\end{array}$ & $\begin{array}{c}\text { phase 2 } \\
(4 \text { à } 8 h)\end{array}$ & $\begin{array}{c}\text { différence entre } \\
\text { phases de culture }\end{array}$ \\
\hline 1 & $-(a)$ & ND(b) & 1 & 1 & NS \\
2 & + & 5 & 7 & 6 & NS \\
& - & ND & 74 & 86 & NS \\
4 & + & 95 & 82 & 88 & S \\
& - & ND & 17 & 10 & NS \\
$\geq 6$ & + & 0 & 8 & 6 & NS \\
& - & ND & 8 & 3 & S \\
\hline
\end{tabular}
(a) :,-+ : respectivement avant et après dispersion $30 \mathrm{~s}$ à l'Ultra-Turrax.
(b) : ND = non déterminé.
(c) : NS, S : différence de moyenne respectivement non significative ot significative à $P=0,05$. 


\section{Niveaux de population dans le lait}

Le Tableau II indique le niveau des bactéries lactiques dans le lait en début et après $24 \mathrm{~h}$ de culture. On observe, dans l'ensemble, une faible dispersion des résultats, ce qui se manifeste par des coefficients de variation bien inférieurs à $10 \%$. Cela signifie, en particulier, une bonne reproductibilité des expériences, tout au moins en ce qui concerne le niveau de population des bactéries lactiques dans le lait. Bien que le test de Bartlett (Snedecor \& Cochran, 1957) n'ait pas permis de mettre en évidence une hétérogénéité significative des variances, on notera cependant que celles-ci ne sont pas dans des rapports voisins : de loin la plus forte, est celle qui correspond au début de la culture de la souche prtCela est dû essentiellement à ce que, dans le premier essai, le niveau d'inoculum était d'environ une unité de log inférieur au niveau des autres essais. Si
I'on élimine ce résultat, on obtient une reproductibilité aussi bonne que dans les autres déterminations.

\section{Vitesse de croissance}

La Fig. 1 présente les résultats d'un essai effectué avec la souche prt -. On observe un assez bon alignement des points correspondant aux résultats obtenus entre 1 et $6 \mathrm{~h}$, ce qui permet de penser que la phase exponentielle de croissance s'est déroulée dans cet intervalle de temps. Le point initial ne paraît pas appartenir à cet ensemble. II est situé au-dessus de la droite, ce qui pourrait signifier que la culture a débuté avec une phase d'adaptation. On peut conclure de l'observation de cette situation dans 4 essais sur 6, que le mode de sélection des cultures-mères est inapproprié : la phase de croissance exponentielle de ces

Tableau II. Niveaux atteints par Lactococcus lactis subsp. lactis (souche protéolytique CNRZ 1076 et variant non protéolytique, $\mathrm{CNRZ} 1075$ ) au cours de leur culture à $30^{\circ} \mathrm{C}$ dans du lait écrémé reconstitué (logarithme du nombre de ufc/ml).

\begin{tabular}{ccccc}
\hline Essai & \multicolumn{2}{c}{$\begin{array}{c}\text { Souche non protéolytique } \\
\text { début de } \\
\text { culture }\end{array}$} & $\begin{array}{c}\text { Souche protéolytique } \\
\text { après } \\
24 \mathrm{~h}\end{array}$ & $\begin{array}{c}\text { sébut de } \\
\text { culture }\end{array}$ \\
$24 \mathrm{~h}$ \\
\hline 1 & 5,1 & 8,6 & 5,8 & 9,4 \\
2 & 5,9 & 8,6 & 6,1 & 9,4 \\
3 & 5,9 & 8,9 & 6,4 & 9,6 \\
4 & 6,0 & 9,1 & 6,1 & 9,6 \\
5 & 6,1 & 9,1 & 6,1 & 9,6 \\
6 & 6,2 & 8,9 & 6,2 & 9,6 \\
moyenne & $5,9 \pm 0,4$ & $8,9 \pm 0,2$ & $6,1 \pm 0,2$ & $9,5 \pm 0,1$ \\
variance & 0,15 & 0,05 & 0,04 & 0,01 \\
coefficient de & & 2,6 & 3,1 & 1,1 \\
variation (\%) & 6,6 & & & \\
\hline
\end{tabular}


cultures a sans doute été dépassée, ce qui entraîne habituellement un retard dans la culture suivante. En effet, il est bien connu (Lawrence et al., 1976), et nous l'avons vérifié par ailleurs pour cette même souche, que les variants prt - des streptocoques lactiques coagulent le lait très longtemps après $y$ avoir atteint leur niveau maximum de population.

L'analyse de régression fournit, pour la pente de la droite, une valeur de 0,47 unité de log de ufc par h (soit un taux de croissance de $1,56 \mathrm{~h}^{-1}$, ou un temps de génération de $38 \mathrm{~min}$ environ). L'écart type $s_{b}$ de la pente était de 0,026 (unité de $\log / \mathrm{h}$ ), ce qui donne un encadrement de la pente de $\pm 0,08$ (unité de log/h), soit une probabilité de $95 \%$ que la valeur exacte de celle-ci soit comprise entre 0,39 et 0,55 unité de $\mathrm{log} / \mathrm{h}$ (soit un temps de génération compris entre 33 et $46 \mathrm{~min}$ ). C'est une marge d'incertitude assez importante $(17 \%)$, mais comme on peut le voir sur le Tableau III les valeurs des

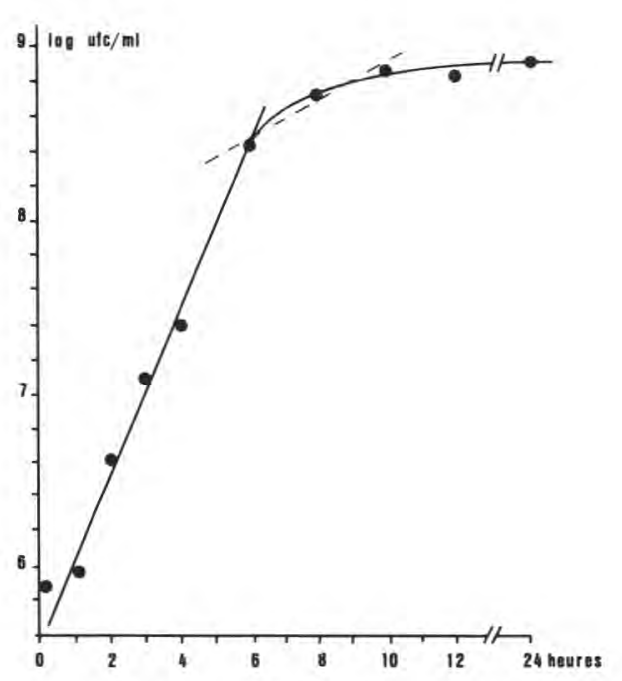

Fig. 1. Evolution de Lactococcus lactis subsp. lactis (souche protéase-négative CNRZ 1075) au cours de sa culture à $30^{\circ} \mathrm{C}$ dans du lait écrémé reconstitué. pentes obtenues au cours des 5 autres essais sont en réalité beaucoup moins dispersées qu'on pouvait le craindre au vu de l'intervalle de confiance d'une seule pente. Seul un essai sur 6 (le $5^{e}$ ), aurait permis, avec une valeur de $s_{b}$ de 0,011 , de déterminer la pente avec la même précision que la moyenne des 6 essais.

La Fig. 2 montre un exemple des résultats obtenus avec la souche $\mathrm{prt}^{+}$. Comme précédemment, nous avons estimé, en première approximation, que les points étaient suffisamment bien alignés, dans l'intervalle de temps s'étendant de 0 à $8 \mathrm{~h}$, pour considérer que la culture $y$ était en phase exponentielle. Au cours de cette période, la souche croît avec une vitesse comprise à $95 \%$, entre 0,36 et 0,44 unité de $\mathrm{log} / \mathrm{h}$ (soit un temps de génération compris entre 41 et $50 \mathrm{~min}$ ). C'est une estimation meilleure que dans l'exemple pris pour la souche prt -. En effet, la marge d'erreur ne représente plus que $10 \%$ environ de la

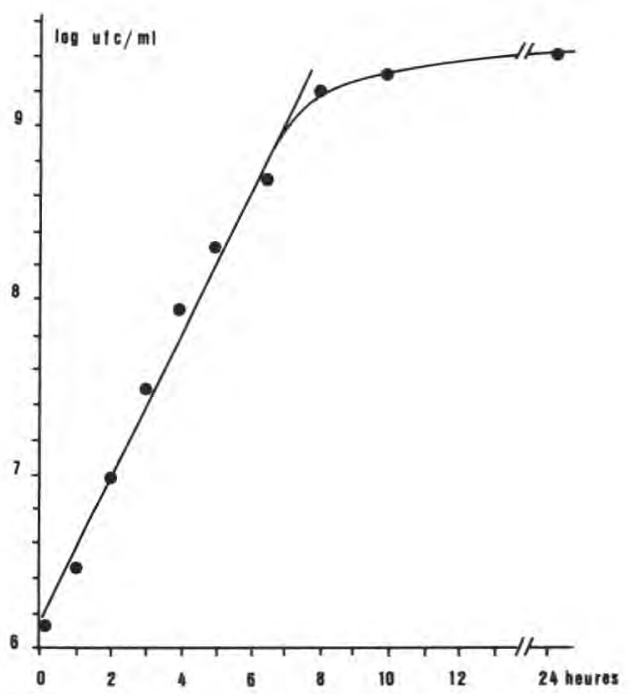

Fig. 2. Evolution de Lactococcus lactis subsp. lactis (souche protéase-positive CNRZ 1076) au cours de sa culture à $30^{\circ} \mathrm{C}$ dans du lait écrémé reconstitué. 
valeur estimée. Les résultats des autres essais avec cette souche ont été traités de la même manière. On observe (Tableau III) que dans l'ensemble, l'estimation de chaque pente est plus précise que pour la souche prt - (valeurs de l'écart type $s_{b}$ des différentes estimations généralement plus faibles et encadrement plus étroit de chaque estimation). On notera toutefois, que l'écart-type de régression $s_{y, x}$ est généralement plus élevé, ce qui est l'indication (peu vraisemblable) d'une moins bonne fidélité des mesures ou celle, plus plausible, d'un moins bon ajustement à la droite de régression. Cela nous oblige à poser le problème de la validité de cet ajustement et par conséquent, de savoir si les bactéries sont réellement en phase exponentielle de croissance, dans l'intervalle de temps délimité au vu de la représentation graphique des résultats.

Tableau III. Caractéristiques de la phase de croissance apparemment exponentielle de Lactococcus lactis subsp. lactis (souches CNRZ 1075, prt-, et 1076, $\mathrm{prt}^{+}$) en culture à $30^{\circ} \mathrm{C}$ dans du lait écrémé reconstitué.

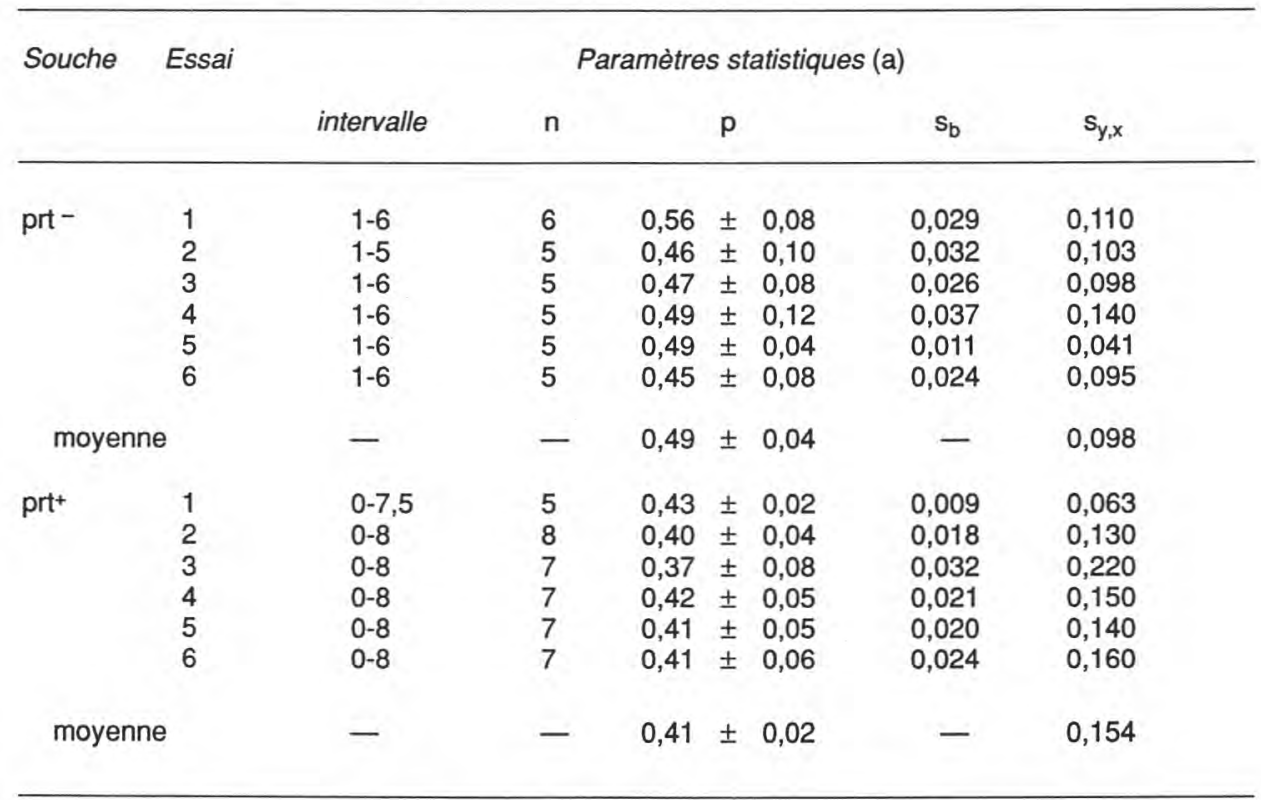

(a) : intervalle = période $(e n \mathrm{~h})$ pendant laquelle la souche est supposée en phase exponentielle de croissance $n=$ nombre de points $p=$ pente de la droite de régression (unité de $\log / \mathrm{h}$ ).

$s_{b}=$ écart type de la pente (unité de log/h)

$s_{y, x}=$ écart type résiduel (unité de log/h). 


\section{Validité de l'ajustement à une droite}

Dans le cas d'une croissance vraiment exponentielle, les écarts entre les nombres de bactéries observées et les nombres prédits par régression devraient être dus essentiellement à l'ensemble des erreurs de mesure. Celles-ci comprennent essentiellement le manque de fidélité de la méthode de mesure proprement dite (variations entre numérations effectuées sur le même échantillon) et la différence réelle entre deux prises d'essai au même instant sur la culture (manque d'homogénéité de celle-ci). Dans une expérience complémentaire à la présente étude, mais dans laquelle la culture était en tubes, nous avons pris, toutes les heures de la première à la $8 \mathrm{e}$, deux tubes, sur chacun desquels nous avons effectué deux séries de dilutions et un ensemencement des boîtes de Petri à partir de chaque série de dilutions. L'analyse de variance sur les logarithmes des nombres de colonies a fait apparaître un écart type de la variabilité entre tubes examinés au même moment égal à 0,10 , significativement supérieur à l'écart type de l'erreur sur le dénombrement, égal à 0,05 . Ce dernier est tout à fait en accord avec les résultats de divers auteurs (Gilchrist et al., 1973 et 1977; Campbell \& Gilchrist, 1973; Donnely et al., 1976; Peeler et al., 1977; O'Connor \& Fleming, 1979; Catteau et al. 1981; Zipkes et al., 1981) qui ont trouvé que l'erreur de répétabilité de l'ensemenceur Spiral était généralement compris entre 0,04 et 0,06 unité de log. Les valeurs de $s_{y, x}$ supérieures à 0,10 dans les analyses de régression traduisent donc, soit un manque d'homogénéité marqué de la culture (ce qui est peu vraisemblable pour des essais réalisés en flacons, avec agitation avant la prise d'essai) ou soit un mauvais ajustement à la droite de régression. $\mathrm{Ce}$ défaut d'ajustement à une droite peut être dû à deux causes principales : la première est que la phase exponentielle est peut-être plus courte qu'il n'apparaît sur la représentation graphique des résultats. Dans ce cas, on peut s'attendre à une déviation systématique, par rapport à la droite de régression, des premiers et des derniers points de l'intervalle. La deuxième cause d'un mauvais ajustement peut provenir du fait que, pour des raisons inhérentes aux bactéries lactiques et au lait comme milieu de culture, la vitesse de croissance cellulaire n'est pas uniforme pendant la phase apparemment exponentielle de croissance, mais varie sous l'effet de divers facteurs. C'est ce qui peut se produire, par exemple, par suite d'un allongement des chaînes de coccien cours de culture, de l'abaissement du pH du lait, ou de l'épuisement du milieu en un nutriment particulier, chacun de ces facteurs ayant pour effet, réel ou apparent, de ralentir la vitesse de croissance des bactéries lactiques. II est difficile de mettre en évidence un tel changement de vitesse de croissance au cours d'une seule expérience, en raison du manque de précision des résultats de dénombrements microbiens. En revanche, une déviation systématique, par rapport à la droite de régression, de points particuliers, peut être mise en évidence par l'analyse statistique des "résidus». Cela consiste d'abord à calculer, pour chaque essai, les écarts ("résidus") à la droite de régression pour chaque temps de culture; on calcule ensuite la moyenne algébrique des écarts correspondant au même temps de culture de chaque essai, ainsi que l'intervalle de confiance de cette moyenne. Cela permet de voir si ces moyennes sont ou non significativement différentes de zéro.

La Figure 3 (a) montre que pour la souche prt -, l'ajustement à une droite, dans l'intervalle de temps de 1 à $6 \mathrm{~h}$, est une hypothèse acceptable. En effet, la 
moyenne des résidus, pour chaque temps de culture dans cet intervalle, n'est pas significativement différente de zéro. On remarquera, en revanche, que les écarts au temps $t=0$ (non compris dans l'intervalle sur lequel on a calculé la droite de régression) sont significativement supérieurs à zéro, ce qui signifie que les points expérimentaux étaient, en général,
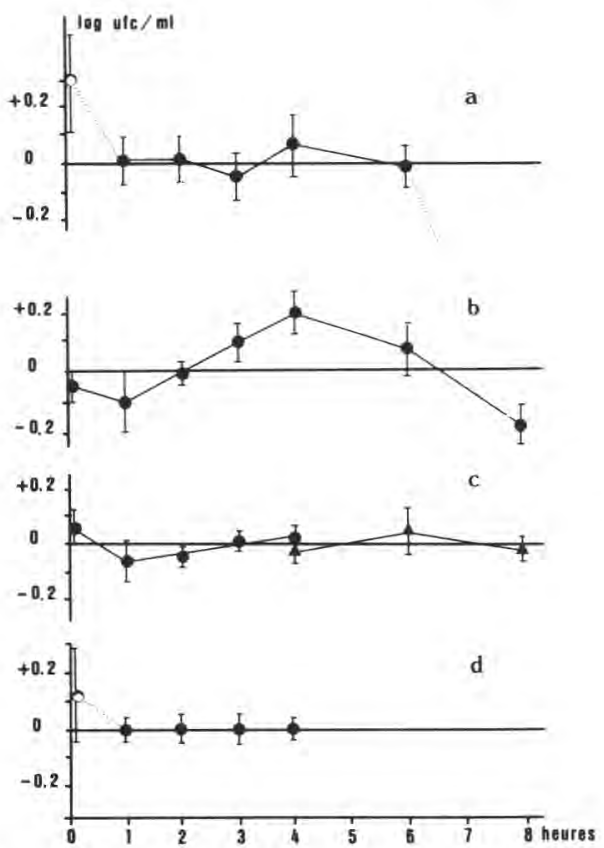

Fig. 3. Moyennes algébriques des écarts à la droite de régression pour les différentes phases exponentielles de croissance de Lactococcus lactis subsp. lactis (souche protéase-négative CNRZ 1075 et souche protéase-positive CNRZ 1076) cultivées à $30{ }^{\circ} \mathrm{C}$ dans du lait écrémé reconstitué (moyenne de 6 essais). (a) : souche CNRZ 1075. Symbole creux : points non inclus dans l'analyse de régression. (b) : souche CNRZ 1076. Modèle comportant une seule phase exponentielle. (c) : comme pour (b). Modèle comportant deux phases exponentielles, l'une de 0 à $4 \mathrm{~h}(--)$, l'autre de 4 à $8 \mathrm{~h}(-\mathbf{A}-)$. (d) : comme pour (c) mais premier intervalle excluant le point correspondant $t=0$. au-dessus de la droite de régression. Cela peut être interprété comme la preuve que la culture nécessitait une phase d'adaptation. A l'inverse, les écarts après $8 \mathrm{~h}$ de culture (hors intervalle de calcul de la droite de régression) étaient largement inférieurs à zéro (moyenne égale à - 0,85 $\pm 0,38$ unité de log), ce qui montre qu'à ce moment, la culture était, comme on pouvait le voir sur les courbes, en dehors de sa phase exponentielle. II est à noter que la limitation de l'intervalle de croissance apparemment exponentielle de 1 à $4 \mathrm{~h}$, ne changeait rien aux moyennes des résidus, pas plus que l'élimination des résultats du premier essai.

La Fig. 3 (b) montre que pour la souche $\mathrm{prt}^{+}$, l'hypothèse d'une phase exponentielle uniforme de zéro à $8 \mathrm{~h}$ peut être rejetée. De plus, l'analyse des résidus indique que les écarts moyens à la droite de régression suivent une évolution régulière en fonction du temps. La forme de cette évolution suggère que la phase de croissance de cette souche se décompose, en fait, en deux phases vraisemblablement exponentielles, l'une de 0 à 4 h, l'autre de 4 h à 8 h. La Fig. 3 (c) montre que cette hypothèse est tout à fait recevable, puisque les écarts moyens à la droite de régression, pour chaque temps de culture, ne sont pas significativement différents de 0 . Cependant, puisque la moyenne des écarts au temps $t=0$ est positive, on peut penser que comme pour la souche prt, la culture nécessite un temps d'adaptation avant d'entrer en phase exponentielle. L'analyse des résidus, pour les calculs des paramètres de croissance dans l'intervalle de temps de 1 à $4 \mathrm{~h}$, renforce cette hypothèse, en donnant des écarts moyens beaucoup plus proches de 0 (Fig. 3 d).

Le Tableau IV donne, finalement, les caractéristiques de la croissance de la souche $\mathrm{prt}^{+}$en prenant, cette fois-ci pour 
hypothèse, l'existence de deux phases exponentielles différentes, la première de 1 à $4 \mathrm{~h}$, la seconde de 4 à $8 \mathrm{~h}$. Par souci d'allègement, on n'a pas présenté, dans ce tableau, les valeurs de $s_{b}$. On peut aisément les retrouver, car elles ont servi au calcul de l'intervalle de confiance des valeurs de pente. On observera tout de suite que les valeurs de $s_{y, x}$ sont, pour la plupart, de l'ordre de l'erreur de la méthode de dénombrement. Dans les deux cas où elle dépasse 0,1 unité de log, la culture était dans sa $2^{e}$ période de croissance; il est possible que le point à $\mathrm{t}=8 \mathrm{~h}$ appartenait déjà à la phase de ralentissement de la culture. C'est le genre de situation illustrée sur la Fig. 1 par le trait discontinu.

II est remarquable que la valeur moyenne de la pente de la première phase de croissance exponentielle de la souche $\mathrm{pr}^{+}$ne diffère plus significa- tivement de celle de la souche prt -. On observera aussi que les estimations de cette pente sont, dans l'ensemble, individuellement plus précises que celles de la souche prt - . Cela pourrait être dû à ce que seulement 4 points ont été retenus pour leur calcul, au lieu de 5 pour la souche prt -, d'où un ajustement plus aisé à une droite. En fait, le calcul des pentes et des valeurs de $s_{y, x}$ sur le même intervalle de 1 à $4 \mathrm{~h}$ pour la souche prt ne changeait rien : on a trouvé une moyenne de pente des 6 essais de 0,49 \pm 0,04 unité de $\log / \mathrm{h}$, et 5 sur 6 des valeurs des $s_{y, x}$ proches de 0,10 (moyenne égale à 0,098 ). On doit en conclure qu'un ajustement à une droite est moins approprié pour la souche prt-que pour la souche $\mathrm{prt}^{+}$. La raison de cette divergence de comportement des deux souches en culture dans le lait est actuellement à l'étude.

Tableau IV. Caractéristiques de la phase de croissance exponentielle de la souche CNRZ 1076 (protéolytique) dans un modèle diphasique.

\begin{tabular}{|c|c|c|c|c|c|c|c|c|c|}
\hline \multirow[t]{2}{*}{ Essai } & \multicolumn{5}{|c|}{ Phase 1} & \multicolumn{4}{|c|}{ Phase 2} \\
\hline & intervalle & $n$ & & $p$ & $s_{y, x}$ & intervalle & $n$ & $p$ & $s_{y, x}$ \\
\hline 1 & $1-7,5$ & 4 & $0,42 \pm$ & $\pm 0,03$ & 0,050 & - & & NO & - \\
\hline 2 & $1-4$ & 4 & $0,49 \pm$ & $\pm 0,02$ & 0,021 & $5-8$ & 3 & $0,30 \pm 0,08$ & 0,041 \\
\hline 3 & $1-4$ & 4 & $0,53 \pm$ & $\pm 0,02$ & 0,016 & $4-8$ & 3 & $0,23 \pm 0,11$ & 0,069 \\
\hline 4 & $1-4$ & 4 & $0,55 \pm$ & $\pm 0,07$ & 0,045 & $4-8$ & 3 & $0,33 \pm 0,01$ & 0,008 \\
\hline 5 & $1-4$ & 4 & $0,50 \pm$ & $\pm 0,09$ & 0,061 & $4-8$ & 3 & $0,33 \pm 0,15$ & 0,098 \\
\hline 6 & $1-4$ & 4 & $0,51 \pm$ & $\pm 0,03$ & 0,020 & $4-8$ & 3 & $0,31 \pm 0,22$ & 0,141 \\
\hline Moyenne & - & - & $0,50 \pm$ & $\pm 0,05$ & 0,036 & - & - & $0,30 \pm 0,05$ & 0,071 \\
\hline
\end{tabular}

$n$ : nombre de points expérimentaux.

- (voir Tableau III pour les autres unités de mesures).

NO: non observé. 


\section{DISCUSSION GÉNÉRALE ET CONCLUSION}

Au cours de cette étude, une relation constante a été obtenue entre le nombre de cellules dans le lait et le nombre de colonies sur le milieu gélosé, cela grâce au traitement des cultures par I'Ultra Turrax, entraînant une dispersion très poussée des agrégats cellulaires des souches choisies. L'efficacité d'une méthode similaire de dispersion des amas cellulaires a été établie pour d'autres souches de streptocoques lactiques par Martley (1972), et l'absence d'effet de ce traitement sur la viabilité des cellules a été montrée par ce même auteur et par d'autres (Lee \& Calcott, 1976). On peut donc être assuré de la validité de la méthode de dénombrement par formation de colonies pour l'étude de croissance de nos deux souches de streptocoque lactique. En particulier, on peut rejeter l'hypothèse que le changement de pente de la souche $\mathrm{prt}^{+}$ après $4 \mathrm{~h}$ de culture, est dû à un allongement progressif des chainettes, entraînant une diminution apparente de la vitesse de croissance de cette souche.

Par ailleurs, on a supposé que la culture des bactéries lactiques dans le lait comportait une phase exponentielle relativement longue (plusieurs $h$ ), au cours de laquelle la vitesse de croissance restait constante. L'objectif implicite de l'étude était de déterminer cette vitesse de croissance, et de voir dans quelle mesure la méthode de dénombrement affectait la précision de cette détermination. En d'autres termes, nous avons utilisé le modèle conceptuel classique de Monod (1949), en postulant une relation constante entre la biomasse et le nombre de cellules :

$$
d x / d t=m x
$$

$x$ étant la biomasse produite, $d x / d t$ la vitesse instantanée de cette production et $\mathrm{m}$, le taux de croissance spécifique de la souche utilisée dans le milieu donné. On a supposé que $m$ était constant dans l'intervalle de temps où les points expérimentaux paraissaient alignés et qu'il existait une relation constante entre la biomasse et le nombre de cellules pendant toute la durée de la culture (croissance équilibrée). C'est évidemment une approche très simplifiée de la croissance microbienne en milieu non renouvelé. Cependant, compte-tenu de la précision de la méthode de dénombrement utilisée, une déviation importante, par rapport au modèle simple que nous avons adopté, n'a pas été mise en évidence pour la souche protéolytique, au moins dans l'intervalle de temps de 1 à 4 h. En revanche, dans ce même intervalle, l'écart type de régression, pour la souche protéase-négative, était significativement plus élevé que pour la souche protéase-positive. Nous avons attribué ce fait à un défaut d'ajustement des points de cet intervalle à la droite de régression, donc au modèle mathématique décrivant la croissance de cette bactérie. II est possible qu'un modèle plus élaboré que celui que nous avons utilisé aurait permis d'avoir un ajustement plus étroit. Cela est vrai aussi pour la souche $\mathrm{prt}^{+}$dans sa $2^{\mathrm{e}}$ phase de croissance. Ce problème fait l'objet d'une autre étude.

Si l'incertitude que le modèle mathématique d'interprétation des résultats peut être l'une des causes d'imprécision des mesures de vitesse de croissance des bactéries lactiques dans le lait, une autre cause demeure : le manque de précision de la méthode de mesure des concentrations cellulaires. Elle affecte aussi bien l'écart type de régression que l'écart type de la pente de la droite de régression, puisque ce dernier est lié au premier par la relation : 


$$
s_{b}=s_{y, x} / \sqrt{\sum\left(t_{i}-t^{-}\right)^{2}}
$$

où $t_{i}$ est une valeur quelconque de l'échelle des temps et $t$, la moyenne de ces valeurs. Si l'on considère une phase de croissance réellement exponentielle, l'écart type de régression sera le même, quelle que soit la longueur de cette phase, puisque seule l'erreur de mesure explique la valeur de $s_{y, x}$. Par conséquent, plus l'échelle de temps est longue, pour un même nombre d'échantillons prélevés au cours de la culture, plus la valeur de $s_{b}$ sera basse, parce que, dans le $2^{e}$ terme de l'équation ci-dessus, le dénominateur augmente. C'est ce qui explique les faibles valeurs de $s_{b}$ des pentes de la souche prt+, dans le Tableau III, alors que les valeurs de $s_{y, x}$ sont élevées. A l'inverse, en réduisant l'intervalle de temps de la première phase de cette souche, pour être sûr qu'elle soit exponentielle, on a diminué considérablement l'écart type de régression, mais pas proportionnellement l'intervalle de confiance des pentes. Cela est dû au fait que l'on a simultanément augmenté l'écart type $s_{b}$ de la nouvelle pente, pour la raison que l'on a indiqué plus haut, et la valeur critique de $t_{0,05}$ servant à calculer cet intervalle de confiance (Snedecor \& Cochran, 1957). Cette valeur $t_{0,05}$ dépend en effet du nombre de degrés de liberté (nombre de points expérimentaux moins 2) : elle passe de 2,571 avec 7 points (5 degrés de liberté), comme dans le Tableau II à 4,303 avec seulement 4 points (résultats du Tableau IV, $1^{\text {re }}$ pente). Avec 3 points (résultats du Tableau IV, $2^{\mathrm{e}}$ pente), elle atteint 12,706 ! II résulte de cela qu'une estimation individuelle de vitesse de croissance basée sur un faible nombre de points expérimentaux peut paraître imprécise (large intervalle de confiance), alors que des expériences répétées dans des conditions identiques, confirment l'exactitude de la première estimation.
C'est un peu ce qui s'est produit dans notre étude.

En conclusion, on peut retenir de cette discussion générale, que pour avoir le maximum de précision dans l'estimation d'une vitesse de croissance cellulaire basée sur la méthode de numération par formation de colonies, il faut agir sur trois plans :

- augmenter la précision des mesures, en réduisant le risque d'erreur liée aux prises d'essai (manque d'homogénéité du milieu de culture) et en augmentant la précision des numérations par des ensemencements multiples (par exemple, une numération en quadruple devrait permettre une diminution de moitié de l'écart type de régression, et du même coup, celui de l'intervalle de confiance d'une valeur de pente),

- augmenter la fréquence des prélèvements, ce qui permet à la fois de délimiter avec plus d'assurance la phase exponentielle de croissance et d'avoir un plus grand nombre de points pour le calcul de la pente permettant de diminuer l'intervalle de confiance de cette détermination,

- répéter l'expérience (au moins trois fois), afin de se prémunir d'un défaut éventuel de reproductibilité de ce genre d'étude.

C'est évidemment un travail très important. II est donc nécessaire de rechercher une méthode de numération aussi sensible que le dénombrement des colonies, mais plus simple à mettre en œuvre, plus économique et donnant des résultats plus rapidement. II faut également optimiser le plan expérimental, de façon à réduire au minimum le nombre de prises d'essai dans chaque expérience. Les informations que nous avons obtenues au cours de cette étude seront très utiles pour réaliser ce dernier objectif. 


\section{RÉFÉRENCES}

Anonyme (1985) Définition et évaluation de la précision globale des méthodes indirectes d'analyse du lait - Application au calibrage et au contrôle. Fédération Internationale de Laiterie, Norme provisoire 128

Anonyme (1986) Validation of the publication of new names and new combinations previously effectively published outside the IJSB. Inter. J. System. Bacteriol. 36, 354-356

Breheny S., Kanasaki M., Hillier A.J. \& Jago G.R. (1975) Effect of temperature on the growth and acid production of lactic acid bacteria. II. The uncoupling of acid production from growth. Aust. J. Dairy Technol. 30, 145148

Catteau M., Auby J.C. \& Catsaras M. (1981) Système spiral et analyse bactériologique des plats cuisinés. Rec. Méd. Vét. 157, 735-739

Campbell J.E. \& Gilchrist J.E. (1973) Spiral plating technique for counting bacteria in milk and other foods. Develop. Ind. Microbiol. 14, 95-102

Cousins C.M., Pettipher G.L., McKinnon C.H. \& Mansell R. (1979) A rapid method for counting bacteria in raw milk. Dairy Ind. Int. $48,45-50$

Donnelly C.B., Gilchrist J.E., Peeler J.T. \& Campbell J.E. (1976) Spiral plate count method for the examination of raw and pasteurized milk. Appl. Environ. Microbiol. 32, 21-27

Fisher R.A., Thornton H.G. \& MacKenzie W.A. (1922) The accuracy of the plating method of estimating the density of bacterial populations. Ann. Appl. Biol. 9, 325-359

Gilchrist J.E., Campbell J.E., Donnelly C.B., Peeler J.T. \& Delaney J.M. (1973) Spiral plate method for bacterial determination. Appl. Microbiol. 25, 244-252

Gilchrist J.E., Donnelly C.B., Peeler J.T. \& Campbell J.E. (1977) Collaborative study comparing the spiral plate and aerobic plate count methods. J. Assoc. Off. Anal. Chem. 60, 807-812

Hobbie J.E., Daley R.J. \& Jasper S. (1977) Use of nucleopore filters for counting bacteria by fluorescence microscopy. Appl. Environ. Microbiol. 33, 1225-1228
Kanasaki M., Breheny S., Hillier A.J. \& Jago G.R. (1975) Effect of temperature on the growth and acid production of lactic acid bacteria. I. A rapid method for the estimation of bacterial populations in milk. Aust. J. Dairy Technol. 30, 142-144

Kouomegne R., Bracquart P. \& Linden G. (1984) Application d'un réactif de transparisation du lait au dénombrement de bactéries. Lait 64, 418-435

Lawrence R.C., Thomas T.D. \& Terzaghi B.E. (1976) Reviews of the progress of Dairy Science : cheese starters. J. Dairy Res. 43, 141-193

Lee S.K. \& Calcott P.H. (1976) Viability determination in chain-forming bacteria. Lab. Pract. 25, 77-79

Martley F.G. (1972) The effect of cell numbers in streptococci chains on plate-counting. N.Z. J. Dairy Sci. Technol. 7, 7-11

Monod J. (1949) The growth of bacterial cultures. Ann. Rev. Microbiol. 3, 371-394

O'Connor F. \& Fleming M.G. (1979) An evaluation of the spiral count method in grading farm milk supplies. Ir. J. Food Sci. Technol. 3, 11-17

Peeler J.T., Gilchrist J.E., Donnelly C.B. \& Campbell J.E. (1977) A collaborative study of the spiral plate method for examining milk samples. J. Food Prot. 40, 462-464

Piton C. \& Richard J. (1983) Influence de l'agitation des échantillons de lait cru sur les résultats de dénombrement de trois groupes d'intérêt technologique. Lait 63, 405-415

Richard J. (1980) Influence de l'agitation du lait cru sur les résultats de dénombrement de sa flore totale à l'aide d'une anse calibrée. Lait 60 , 211-225

Rodrigues U.M. \& Kroll R.G. (1985) The direct epifluorescent filter technique (DEFT) : increased selectivity, sensitivity and rapidity. $J$. Appl. Bacteriol. 59, 493-499

Snedecor G.W. \& Cochran W.G. (1957) Statistical methods. The lowa State University Press, Ames, lowa, USA

Tatini S.R., Dabbah R. \& Olson Jr J.C. (1967) Comparison of plate loop and agar plate methods for bacteriological examination of manufacturing grade of raw milk. J. Milk Food Technol. 30, 112-115 
Terzaghi B.E. \& Sandine W.E. (1975) Improved medium for lactic streptococci and their bacteriophages. Appl. Microbiol. 29, 807-813

Turner K.W. \& Thomas T.D. (1975) Uncoupling of growth and acid production in lactic streptococci. N. Z. J. Dairy Sci. Technol. 10, 162-167

Zipkes M.R., Gilchrist J.E. \& Peeler J.T. (1981) Comparison of yeast and mold counts by spiral, pour, and streak plate methods. J. Assoc. Off. Anal. Chem. 64, 1456-1469 